\title{
Modelagem computacional por elementos finitos de problemas da termoelasticidade bidimensional
}

\author{
Natan Sian das Neves ${ }^{1}$ \\ Programa de Engenharia Civil, COPPE/UFRJ, Rio de Janeiro, RJ \\ Vitor Pancieri Pinheiro \\ Programa de Pós-graduação em Engenharia Mecânica - PPGEM/UFES, Vitória, Brasil.
}

\begin{abstract}
Resumo. Este artigo concentra-se na avaliação numérica de problemas físicos governados pela teoria da termoelasticidade bidimensional linear. As soluções são direcionadas com base nos procedimentos numéricos de elementos finitos de Galerkin. Para tanto, realiza-se uma implementação computacional estruturada desenvolvida em ambiente Matlab. As investigações e os testes numéricos são guiados por casos-testes com soluções analíticas disponíveis na literatura, onde realiza-se uma análise do desempenho do código computacional desenvolvido na previsão do comportamento físico dos problemas estudados. Uma chapa fina confinada sob fluxo de calor constante e um disco circular com aquecimento local são analisados neste trabalho. Em síntese, os resultados numéricos obtidos com o modelo desenvolvido exibem um comportamento satisfatório, atingindo valores coerentes fisicamente de tensão e deslocamento nos elementos estruturais sob ação dos efeitos térmicos.
\end{abstract}

Palavras-chave. Elementos Finitos, Termoelasticidade, Efeitos Térmicos, Código Computacional.

\section{Introdução}

Elementos estruturais (e.g. vigas, pilares, barras, eixos e peças mecânicas) são frequentemente utilizados em projetos industriais e na construção civil, e ao longo da sua vida útil podem ser expostos a diferentes ações e condições adversas, resultando em comportamentos e efeitos físicos inesperados na concepção e análise do sistema estrutural. Dentre as ações de interesse da engenharia estrutural, destaca-se os efeitos da variação de temperatura, presentes em diversas aplicações, por exemplo, na análise de estruturas em condição de incêndio, juntas de dilatação, projetos mecânicos de turbinas e motores a reação e processos industriais para tratamentos térmicos de peças.

O comportamento dos elementos estruturais podem ser descritos por diferentes modelos físicosmatemáticos, sendo papel do usuário escolher o modelo mais adequado para a solução do problema físico. Em inúmeros casos práticos da engenharia, utilizam-se métodos e fórmulas elementares provenientes da resistência dos materiais que proporcionam soluções aceitáveis e seguras. No entanto, é sabido que esses enfoques simplificados não direcionam respostas satisfatórias para avaliação de estruturas com maior nível de complexidade ou configurações físicas não usuais, sendo necessário recorrer aos modelos mais sofisticados da teoria da elasticidade. Todavia, os problemas dessa natureza apresentam dificuldades ao longo do processo de solução via métodos analíticos por conta da complexidade dos problemas físicos, possibilitando encontrar as soluções somente para casos simples. Sendo assim, pode-se recorrer aos métodos aproximados de engenharia, por exemplo, o método das diferenças finitas (MDF), elementos finitos (MEF), elementos de contorno (MEC) e métodos sem malha (meshless).

\footnotetext{
1natan.neves@coppe.ufrj.br

2 vitor.pinheiro1987@gmail.com
} 
Neste cenário, em razão da importância dos efeitos térmicos na resposta mecânica dos elementos estruturais, bem como a visível dificuldade de solução dos problemas da teoria da elasticidade, o presente trabalho busca apresentar as principais ideias acerca da modelagem computacional por elementos finitos em problemas da termoelasticidade bidimensional linear. Um código computacional é desenvolvido, em ambiente Matlab, utilizando uma programação estruturada, onde são implementados os elementos finitos planos do tipo quadrilateral de 4, 8 e 9 nós, e o elemento finito triangular linear de 3 nós. O programa computacional é denominado de NASEN (Numerical Analysis System for Engineering) [3-5]. A experimentação numérica e avaliação de desempenho do programa são direcionados por casos-testes com soluções de referência disponíveis na literatura.

\section{Teoria física-matemática e modelagem numérica}

Na teoria da elasticidade, existem classes de problemas estruturais da engenharia que respeitam certas configurações geométricas e de carregamentos que possibilitam ser analisados em um espaço bidimensional, ou seja, grandezas físicas como tensão, deformação e deslocamento dependem somente de duas variáveis espaciais. Neste cenário, tais classes são conhecidas como estado plano de tensão ou deformação. Vale mencionar que os problemas são desenvolvidos considerando o comportamento do material elástico-linear e a hipótese de pequenos deslocamentos [2].

Neste contexto, considere o elemento infinitesimal com os lados $d x$ e $d y$, submetido às tensões normais $\sigma_{x x}$ e $\sigma_{y y}$ atuando nas direções $x$ e $y$, respectivamente, sendo que essas são perpendiculares às facetas do elemento. A tensão de cisalhamento $\tau_{x y}$ atua paralela em cada faceta do elemento. As condições de equilíbrio aplicadas em um elemento diferencial de um corpo sólido, exigem que as componentes do tensor das tensões, nas direções $x$ e $y$ respeitem a sentença matemática apresentada na equação (1), dita como equação de equilíbrio - representada na forma matricial.

$$
\begin{aligned}
& \nabla_{s}^{T} \underset{\sim}{\sigma}+\underset{\sim}{b}=0 \\
& \underset{\sim}{\varepsilon}=\nabla_{s} u \\
& \underset{\sim}{\sigma}=\underset{\sim}{\sigma} \sigma_{L}+\underset{\sim}{\sigma_{0}}-\sigma_{T} \\
& \mathbf{u}=\bar{u} \quad \text { em } \Gamma_{u} \quad \mathbf{t}=\tau \underset{\sim}{\mathbf{n}} \quad \mathrm{em} \Gamma_{t}
\end{aligned}
$$

Designa-se por $\underset{\sim}{b}$ é o vetor que contém as componentes da força de campo nas respectivas direções cartesianas e $\underset{\sim}{\sigma}$ é o vetor que guarda as tensões planas $\left(\sigma_{x x}, \sigma_{y y}\right.$ e $\left.\sigma_{x y}\right)$. Além disso, têm-se as equações diferenciais cinemáticas ou usualmente conhecidas como as relações de deformaçãodeslocamento. A partir de um elemento diferencial, mostra-se o estado indeformado e deformado do corpo sólido.

Com base nas configurações puramente geométricas e respeitando a hipótese de pequenas deformações, pode-se escrever, na forma matricial, a seguinte relação matemática relacionada ao vetor das componentes de deformação do estado plano, conforme posto na equação (2).

$\mathrm{O}$ vetor $(\underset{\sim}{\varepsilon})$ contém as componentes $\varepsilon_{x x}$ e $\varepsilon_{y y}$, que representam as deformações específicas (ou alongamentos unitários) nas direções $x$ e $y$, respectivamente, e $\gamma_{x y}$ é designada como a deformação angular (ou por cisalhamento). Quando um corpo sofre deformação devido a um carregamento externo aplicado, existe uma relação entre tensão-deformação. Desta forma, assume-se a hipótese que o corpo sofre uma excitação inicial devido uma tensão $\sigma_{0}$ e admite-se também a existência de deformações de origem térmica [1]. Sendo assim, pode-se escrever a seguinte sentença matemática:

Realizando uma investigação acerca da interpretação física dos termos matemáticos presentes na equação (3), sendo que a primeira parcela está relacionada com as tensões associadas à lei de Hooke, a segundo parcela representa as tensões iniciais e, por fim, a terceira parcela é relacionada as 
tensões térmicas, originadas, por exemplo, pela variação de temperatura não uniforme no elemento, uma restrição ao corpo de se expandir livremente ou por uma combinação desses dois efeitos [7].

Por fim, os corpos estão sujeitos a certas condições de restrições de movimento ao longo da sua fronteira. Basicamente, na equação (4) define-se as condições de contorno do tipo essencial e natural, válidas em $\Gamma_{u}$ e $\Gamma_{t}$, respectivamente. Sendo que $\mathbf{t}$ é o vetor de tração no contorno, $\mathbf{n}$ é o vetor normal unitário e $\bar{u}$ é definido como o deslocamento prescrito.

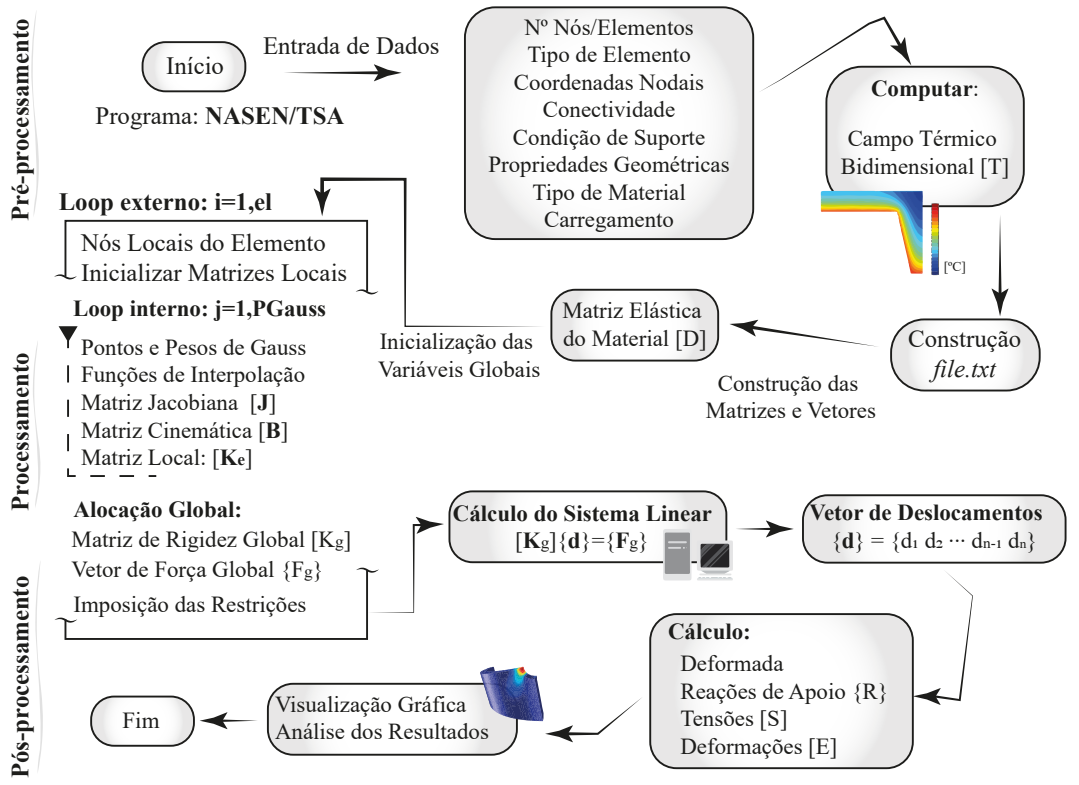

Figura 1: Fluxograma geral do processo de solução do programa computacional

Com base no conhecimento prévio das equações de equilíbrio, cinemáticas e constitutivas no contexto da teoria da elasticidade bidimensional, pode-se aplicar os procedimentos numéricos de elementos finitos. É importante destacar que é possível, alternativamente, realizar o tratamento numérico com base na equação de Navier para sólidos que apresenta os deslocamentos como variável primal [9].

Sendo assim, a partir das premissas do método de resíduos ponderados, após algumas manipulações matemáticas e aplicações dos teoremas do cálculo diferencial-integral, chega-se na formulação variacional fraca para o problema de elasticidade [6], conforme apresentado na equação $(5)$.

$$
\int_{\Omega}\left(\nabla_{s} w\right)^{T} \underset{\sim}{\sigma} d \Omega=\int_{\Gamma}(w)^{T} \mathbf{t} d \Gamma+\int_{\Omega}(w)^{T} \underset{\sim}{b d \Omega}
$$

onde $w$ é a função auxiliar (ou peso). O próximo passo do desenvolvimento dos procedimentos de elementos finitos é substituir o vetor de tensão, com base na lei constitutiva do material, e em seguida, introduz a solução aproximada, escrita com base nas funções de interpolação, na formulação fraca do problema.

A partir das premissas de elementos finitos, pode-se aproximar o campo de deslocamento e a função auxiliar com base na combinação linear entre os coeficientes $\left(u_{j}\right.$ e $\left.w_{i}\right)$ e as funções de interpolação $(\tilde{\mathbf{N}})$, conforme mostrado na equação (6). Além disso, ressalta-se que as funções de interpolação devem ser unitárias nos pontos de análise e nulo nas demais, ou seja, 


$$
\underset{\sim}{u}=\sum_{j=1}^{n} \tilde{\mathbf{N}}_{\mathrm{j}} u_{j} \quad w=\sum_{i=1}^{n} \tilde{\mathbf{N}}_{i} w_{i}
$$

onde $\underset{\sim}{u}$ e $w$ são, respectivamente, os vetores que guardam as componentes, nas direções cartesianas, do deslocamento e da função auxiliar, e a matriz $\tilde{\mathbf{N}}$ contém as funções de forma. Sendo assim, a partir da equação (5) e da equação (6), realizando algumas operações matemáticas, é possível definir o sistema linear resultante, sendo $\Delta$ o vetor de deslocamentos nodais discretos nas direções cartesianas.

$$
[\mathbf{K}]\{\Delta\}=\left\{\mathbf{F}_{c}\right\}+\left\{\mathbf{F}_{t}\right\}+\left\{\mathbf{F}_{B}\right\}+\left\{\mathbf{F}_{t h}\right\}+\left\{\mathbf{F}_{\sigma_{0}}\right\}
$$

Ressalta-se que o termo $\mathbf{F}_{c}$ representa o vetor de forças nodais concentradas da estrutura. Além disso, o vetor de forças nodais térmicas é obtido com base na distribuição bidimensional de temperatura $(T)$ do corpo [10]. Por conta da natureza desacoplada do problema termoelástico estudado, o campo de temperatura pode ser determinado de maneira independente da solução estrutural. No programa desenvolvido, o campo térmico pode ser computado por meio da imposição direta de uma expressão matemática representativa do campo de temperatura atuando no domínio ou pela solução numérica da equação de difusão de calor bidimensional estacionária, conforme posto na equação (8)

$$
\lambda \nabla^{2} T+Q=0
$$

sendo que $\lambda$ é a difusividade térmica do material e $Q$ é a geração interna de calor. Os procedimentos numéricos de elementos finitos aplicados aos problemas de natureza difusiva seguem as mesmas ideias apresentadas anteriormente [6].
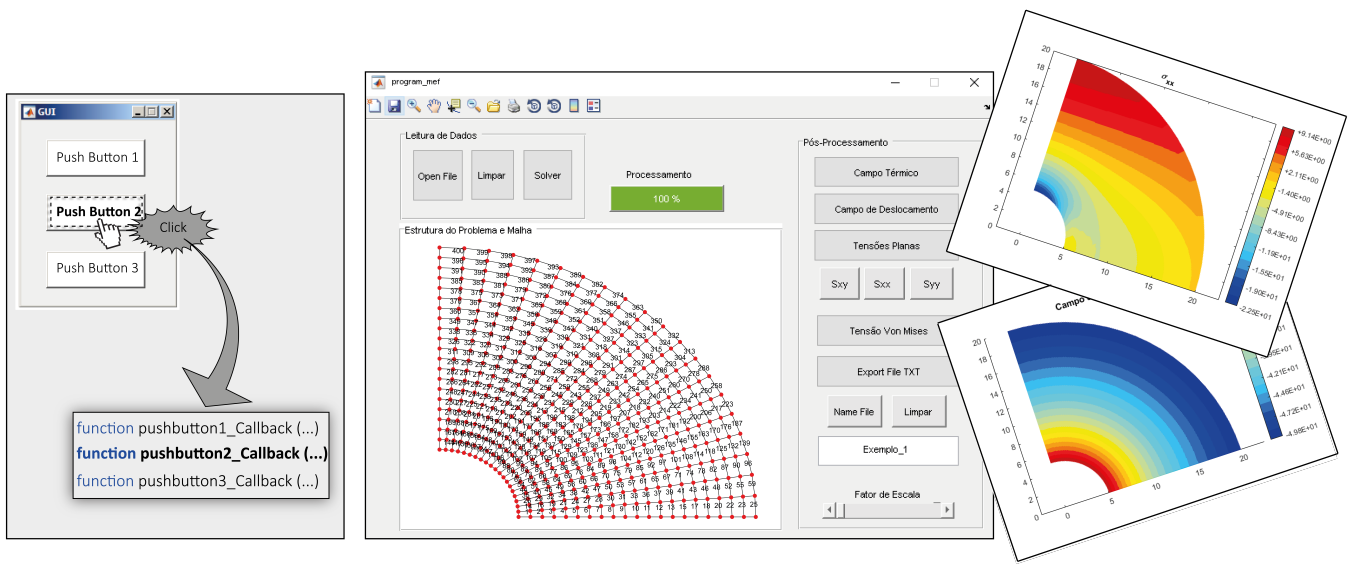

Figura 2: Ideia da programação orientada à eventos e interface gráfica do programa desenvolvido

A implementação computacional é desenvolvida em ambiente Maltab. A escolha dessa linguagem é por conta da simplicidade na programação e pela disponibilidade de diferentes tipos de recursos gráficos. Na Figura 1 apresenta-se um estrutura geral do processo de solução via elementos finitos em problemas da termoelasticidade plana. Para facilitar a aplicação do programa e 
visualização de resultados (pós-processamento) do programa, foi desenvolvido uma interface gráfica simples em ambiente Matlab. A interface gráfica do programa de simulação é ilustrada na Figura 2. Essa ferramenta de cálculo simples e interativa acerca da aplicação de elementos finitos em problemas de termoelasticidade plana, permite ao usuário uma interação direta com o programa via elementos gráficos (botões, slider, menus, etc.). A dinâmica do programa é realizada por eventos, ou seja, as diferentes ações provenientes do usuário sobre elementos gráficos da interface. Cada evento está interligado por uma função denotada Callback.

É possível extrair diferentes informações acerca do problema de maneira direta na interface, por exemplo, o campo térmico, campo de deslocamento, tensões planas e de Von Mises. É importante salientar que com uma interface computacional simples, os usuários podem realizar análises e visualizar os resultados rapidamente. Esses tipos de recursos computacionais são importantes para os docentes/discentes em sala de aula, uma vez que possibilitam um dinamismo e engajamento dos discentes no entendimento dos problemas estudados.

\section{Exemplos numéricos de aplicação}

Primeiramente, analisa-se, por meio de um modelo numérico de elementos finitos, uma chapa quadrada ausente de forças no contorno e sob ação de uma variação linear de temperatura. Em relação às condições de contorno, restringem-se os movimentos nas três extremidades da chapa, conforme exemplificado na Figura 3a.

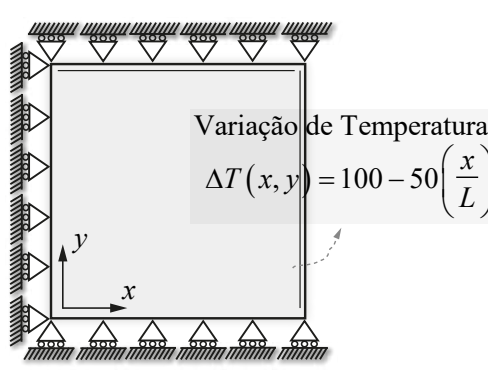

(a)

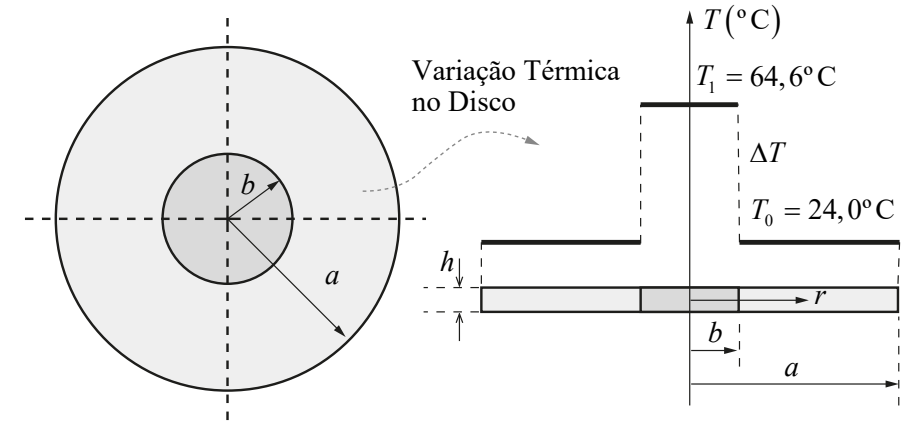

(b)

Figura 3: Casos estudados: (a) chapa quadrada sob variação térmica e (b) disco circular aquecido

A solução analítica do problema pode ser facilmente obtida pelas teorias clássicas, conforme pode ser consultado em Boley e Weiner [1]. Recentemente, Silva, Telles e Santiago [8] apresentam a solução analítica e numérica desse problema, sendo utilizado o método dos elementos contorno. Para a construção da malha computacional, utiliza-se o elemento triangular linear de três nós, sendo realizadas 45 divisões por aresta da chapa, totalizando 5690 elementos finitos e 2936 nós.

São admitidos os seguintes valores hipotéticos para as propriedades físicas e geométricas, onde considera-se um módulo de elasticidade de $2,00 \cdot 10^{8} \mathrm{kPa}$, um coeficiente de expansão térmica igual a $0,001{ }^{\circ} \mathrm{C}^{-1}$, um coeficiente de Poisson de 0,3 e uma chapa com dimensões unitárias. Os valores de tensão normal e de deslocamento da chapa dependem da distribuição de temperatura em torno do domínio da placa. Nas configurações adotadas para o problema, tem-se uma evolução unidimensional de temperatura, variando exclusivamente na direção $x$ da chapa.

Os resultados numéricos são avaliados ao longo do eixo horizontal da chapa, como posto na Tabela 1. Como pode-se verificar, os deslocamentos apresentam baixos níveis de erros percentuais. 
Quando se analisa as tensões na chapa, observa-se que os valores apresentam também boa semelhança com a solução exata, tendo os maiores níveis de erro nas extremidades da chapa devido ao processo de suavização média de tensão.

Tabela 1: Comparação dos resultados numéricos obtidos para tensão normal e deslocamento na chapa com extremidades restringidas

\begin{tabular}{cccccccc}
\hline \multirow{2}{*}{$x(\mathrm{~m})$} & \multirow{2}{*}{$\Delta T\left({ }^{\circ} \mathrm{C}\right)$} & \multicolumn{2}{c}{ Tensão Normal $\left(\mathrm{kN} / \mathrm{m}^{2}\right)$} & \multicolumn{3}{c}{ Deslocamento $(\mathrm{m})$} \\
\cline { 3 - 7 } & & Exata & NASEN & $|\Delta|(\%)$ & Exata & NASEN & $|\Delta|(\%)$ \\
\hline 0,00 & $1,00 \mathrm{E}+02$ & $2,00 \mathrm{E}+07$ & $1,99 \mathrm{E}+07$ & $3,11 \mathrm{E}-01$ & $0,00 \mathrm{E}+00$ & $0,00 \mathrm{E}+00$ & - \\
0,11 & $9,44 \mathrm{E}+01$ & $1,89 \mathrm{E}+07$ & $1,89 \mathrm{E}+07$ & $2,21 \mathrm{E}-03$ & $1,40 \mathrm{E}-02$ & $1,40 \mathrm{E}-02$ & $2,23 \mathrm{E}-03$ \\
0,20 & $9,00 \mathrm{E}+01$ & $1,80 \mathrm{E}+07$ & $1,80 \mathrm{E}+07$ & $1,39 \mathrm{E}-03$ & $2,47 \mathrm{E}-02$ & $2,47 \mathrm{E}-02$ & $8,21 \mathrm{E}-04$ \\
0,31 & $8,44 \mathrm{E}+01$ & $1,69 \mathrm{E}+07$ & $1,69 \mathrm{E}+07$ & $1,13 \mathrm{E}-03$ & $3,73 \mathrm{E}-02$ & $3,73 \mathrm{E}-02$ & $4,07 \mathrm{E}-04$ \\
0,40 & $8,00 \mathrm{E}+01$ & $1,60 \mathrm{E}+07$ & $1,60 \mathrm{E}+07$ & $7,71 \mathrm{E}-04$ & $4,68 \mathrm{E}-02$ & $4,68 \mathrm{E}-02$ & $2,41 \mathrm{E}-04$ \\
0,51 & $7,44 \mathrm{E}+01$ & $1,49 \mathrm{E}+07$ & $1,49 \mathrm{E}+07$ & $9,74 \mathrm{E}-05$ & $5,80 \mathrm{E}-02$ & $5,80 \mathrm{E}-02$ & $1,34 \mathrm{E}-04$ \\
0,60 & $7,00 \mathrm{E}+01$ & $1,40 \mathrm{E}+07$ & $1,40 \mathrm{E}+07$ & $4,78 \mathrm{E}-04$ & $6,63 \mathrm{E}-02$ & $6,63 \mathrm{E}-02$ & $9,21 \mathrm{E}-05$ \\
0,71 & $6,44 \mathrm{E}+01$ & $1,29 \mathrm{E}+07$ & $1,29 \mathrm{E}+07$ & $1,06 \mathrm{E}-03$ & $7,60 \mathrm{E}-02$ & $7,60 \mathrm{E}-02$ & $4,21 \mathrm{E}-05$ \\
0,80 & $6,00 \mathrm{E}+01$ & $1,20 \mathrm{E}+07$ & $1,20 \mathrm{E}+07$ & $2,61 \mathrm{E}-03$ & $8,32 \mathrm{E}-02$ & $8,32 \mathrm{E}-02$ & $8,95 \mathrm{E}-06$ \\
0,91 & $5,44 \mathrm{E}+01$ & $1,09 \mathrm{E}+07$ & $1,09 \mathrm{E}+07$ & $1,68 \mathrm{E}-02$ & $9,15 \mathrm{E}-02$ & $9,15 \mathrm{E}-02$ & $4,83 \mathrm{E}-05$ \\
1,00 & $5,00 \mathrm{E}+01$ & $1,00 \mathrm{E}+07$ & $1,01 \mathrm{E}+07$ & $6,48 \mathrm{E}-01$ & $9,75 \mathrm{E}-02$ & $9,75 \mathrm{E}-02$ & $4,00 \mathrm{E}-03$ \\
\hline
\end{tabular}

Em um segundo momento, estuda-se, numericamente, um caso de uma aplicação da análise de tensão de origem térmica. Sendo assim, considera-se um disco fino de raio $a$ com uma região circular concêntrica aquecida com raio igual $b$, ausente de carregamentos externos aplicados no contorno ou forças de campo, conforme ilustra-se a seguir na Figura 3b. Além disso, as distribuições de temperatura e as tensões são unidimensionais, ou seja, essas são funções das coordenadas radiais do disco.

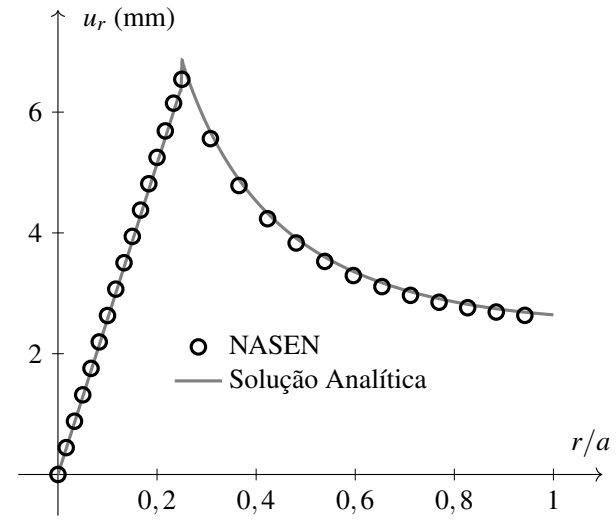

(a)

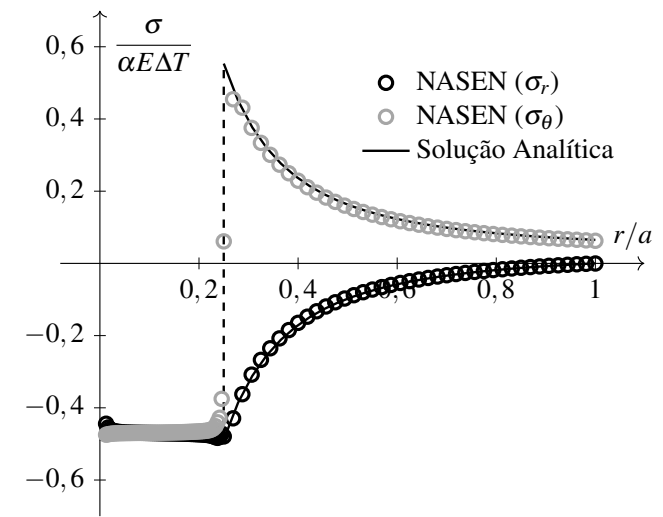

(b)

Figura 4: Comparação entre a solução analítica e numérica para deslocamento radial do disco

No que se refere às propriedades do material da peça estrutural, utiliza-se um módulo de elasticidade igual a $2 \cdot 10^{8} \mathrm{kN} / \mathrm{m}^{2}$, um coeficiente de dilatação térmica de $0,001^{\circ} \mathrm{C}^{-1}$, um coeficiente de Poisson igual a 0,25. Considera-se uma razão entre o raio da região circular aquecida e raio do disco igual a 1/4, adotando o raio do disco igual a $1 \mathrm{~m}$.

A distribuição de temperatura está variando como uma função degrau (ver Figura 3b), fornecendo uma complexidade adicional ao problema por conta da descontinuidade do campo térmico 
na fronteira da região circular centrada aquecida, localizado em $r=b$. A temperatura de referência do disco é mantida constante e igual a $24^{\circ} \mathrm{C}$ e a temperatura do ponto quente local permanece fixada em $64,6^{\circ} \mathrm{C}$, resultando em uma variação térmica de $40,6^{\circ} \mathrm{C}$.

A primeira análise dos resultados é destinada ao campo radial de deslocamento, conforme apresenta a Figura 4a, onde pode-se verificar que os valores obtidos numericamente por meio do método dos elementos finitos apresentam boa concordância em relação aos resultados encontrados com a solução analítica.

Em seguida, são analisados os valores relacionados ao cálculo da tensão radial e circunferencial atuantes no disco, conforme posto na Figura 4b. Deve-se salientar que pelo fato da presença do ponto de descontinuidade no campo térmico do disco, a solução numérica de elementos finitos encontra uma dificuldade próxima à fronteira da região do aquecimento local. Em linhas gerais, o código numérico implementado consegue descrever razoavelmente a variação de tensão no disco.

\section{Conclusões}

Este trabalho buscou realizar uma avaliação numérica acerca do comportamento de problemas da termoelasticidade bidimensional. As soluções dos problemas físicos são direcionados por meio dos procedimentos numéricos de elementos finitos de Galerkin. Os testes são realizados com dois exemplos de aplicação, uma chapa fina confinada sob fluxo de calor constante e um disco circular com aquecimento local, que servem como base para as investigações e a avaliação de desempenho do código computacional de elemento finitos desenvolvido. Ao fim, com base nos resultados numéricos obtidos e as comparações realizadas com as soluções de referência, pode-se concluir que o programa computacional desenvolvido apresentou resultados satisfatórios, possibilitando estimar adequadamente o comportamento físico dos problemas estudados.

\section{Referências}

[1] Boley, B, Weiner, J. Theory of thermal stresses. Dover Edition, Inc.; 1985.

[2] Den Hartog, J. Advanced strength of materials. Dover Publications, Inc.; 1901.

[3] Neves, N. S. Modelo computacional avançado para análise de estruturas sob ação de gradientes térmicos. Dissertação (Mestrado). Universidade Federal do Espírito Santo, Vitória, 2019.

[4] Neves, N. S. Application of the finite element method for two-dimensional thermal analysis of steel-concrete composite structures in fire. In: 18th ENCIT, p. 01 - 06, 2020.

[5] Neves, N. S., Azevedo, M. S., Barcelos, C. B., Silva, V. P., Pierin, I. Estudo térmico de pilares mistos de aço e concreto de seção circular em situação de incêndio. REA, p. 122-140, 2020.

[6] Reddy, J. An introduction to the finite element method. McGraw-hill; 1993.

[7] Ribeiro, H. Análise de estruturas com carregamento térmico utilizando o método dos elementos de contorno. Instituto Militar de Engenhara - IME; 1991.

[8] Silva, A, Telles, J, Santiago, J. Análise termoelástica de placas de Reissner via método dos elementos de contorno. In Simpósio de Mecânica Computacional - SIMMEC 2018.

[9] Timoshenko, S, Goodier, J. Teoria da elasticidade. Guanabara Dois; 1980.

[10] Vila Real, P. Modelação por elementos finitos do comportamento térmico e termo-elástico de sólidos sujeitos a elevados gradientes térmicos. FEUP, 1988. 